www.jmscr.igmpublication.org

Impact Factor 5.84

Index Copernicus Value: 83.27

ISSN (e)-2347-176x ISSN (p) 2455-0450

crossref DOI: https://dx.doi.org/10.18535/jmscr/v5i8.161

Journal Of Medical Science And Clinical Research

\title{
Radiological Response Assessment in High Grade Gliomas: 3DCRT vs IMRT \\ Authors
}

\section{Dr Rahul Loni ${ }^{1}$, Dr Chaitanya $\mathbf{S}^{2}$, Dr Poojar Sridhar ${ }^{3}$, Dr Naveen $\mathbf{T}^{4}$, Dr Ibrahim Khaleel ${ }^{5}$, Dr K.P.R. Pramod ${ }^{6}$, Dr Lokesh $\mathbf{V}^{7}$}

${ }^{1,2}$ Post-Graduate Student, Department of Radiation Oncology, Kidwai Cancer Institute, Bengaluru-29

3,5 Assistant Professor, Department of Radiation Oncology, Kidwai Cancer Institute, Bengaluru-29

${ }^{4}$ Associate Professor, Department of Radiation Oncology, Kidwai Cancer Institute, Bengaluru-29

${ }^{6}$ Department of Radiation Oncology, Kidwai Cancer Institute, Bengaluru-29

${ }^{7}$ Professor and Head, Department of Radiation Oncology, Kidwai Cancer Institutte, Bengaluru-29

Corresponding Author

\section{Dr Chaitanya S}

Post-Graduate Student, Department of Radiation Oncology, Kidwai Cancer Institute, Bengaluru-29 Email: drchaitanya21@gmail.com

\section{ABSTRACT}

Objectives: To compare the radiological response in high grade glioma patients receiving post-operative radiation therapy via 3DCRT /IMRT technique with concurrent and adjuvant temozolomide.

Materials and Methods: Twenty newly diagnosed histologically proven patients of high grade glioma in the age group of 18-70 years, post maximal resection were recruited and all patients received concurrent chemo-irradiation followed by adjuvant chemotherapy. All patients received a dose of 60 Gy in 30 fractions via 3DCRT/IMRT technique along with concurrent and adjuvant temozolomide according to Stupp's regimen. Radiological response was assessed using Cho:NAA ratio in magnetic resonance spectroscopy done at 0,4th and 14th week of radiation therapy. Results: There was no statistically significant difference in the post radiation therapy radiological response with respect to Cho:NAA ratio between the 2 groups suggesting similar outcomes when high grade gliomas are treated with 3DCRT / IMRT technique.

Conclusion: In patients diagnosed with high grade glioma treated by post-operative radiation therapy with concurrent and adjuvant temozolomide, radiation therapy can be delivered either with 3DCRT or IMRT technique without compromise in radiological response to treatment.

Keywords: Intensity Modulated Radiation Therapy (IMRT), 3D Conformal Radiation Therapy (3DCRT), Temozolomide, MRS (Magnetic Resonance Spectroscopy), glioma.

\section{Introduction}

Glioblastoma and other high grade gliomas (anaplastic astrocytoma and anaplastic oligodendroglioma) are the most common primary intracranial tumours and account for more than $30 \%$ of all primary CNS tumours in adults ${ }^{[1]}$. They are associated with a uniformly dismal prognosis despite advances in therapy. These tumours are characterized by resistance to all these therapies and frequently recur quite rapidly within months of any form of treatment ${ }^{[2]}$.

The standard treatment for high grade gliomas starts with maximal safe surgical resection. After surgery, radiotherapy (RT) with concurrent temozolomide followed by adjuvant temozolomide (TMZ) therapy has shown 
significant, although modest, survival benefit over RT alone. In a phase 3 clinical trial conducted by the European Organization for Research and Treatment of Cancer (EORTC) and the National Cancer Institute of Canada (NCI), median overall survival in the chemo-radiotherapy arm was 14.6 months as compared to 12 months in the RT alone arm and the percentage of patients alive at 2 years increased significantly from approximately $10 \%$ to approximately $26 \%$. However, the median progression-free survival even after RT and concurrent temozolomide and adjuvant temozolomide therapy is only 7 months, and in a subset of patients' tumours show aggressive growth despite combined chemo-radiotherapy ${ }^{[3]}$.

The site of the primary tumouris subjected to radiation, chemotherapy and surgical resection. All these cause gross alteration in imaging features which are often nonspecific and inconclusive on conventional MRI sequences. Routine MRI cannot reliably distinguish tumour recurrence or progression from the inflammatory or necrotic changes resulting from radiation necrosis. Radiation necrosis is an end point of radiation therapy and is the worst adverse effect of radiotherapy ${ }^{[4,5,6]}$. The two entities may be distinguished by a brain biopsy, the patient's clinical course or follow-up imaging ${ }^{\text {[7-11] }}$.

20 to $30 \%$ of patients undergoing their first post radiation MRI show increased contrast enhancement that eventually subsides without any change in therapy. This phenomenon, termed pseudo progression, likely results from transiently increased permeability of the tumour vasculature from irradiation, which may have been enhanced by temozolomide and complicates the determination of tumour progression immediately after completion of radiotherapy. This treatment related effect has implications for patient management and may result in premature discontinuation of effective adjuvant therapy ${ }^{[12]}$. While increased contrast enhancement on MRI following chemo-radiation therapy is often interpreted as progression of disease, differentiation of pseudo-progression/treatment induced necrosis from true progression or recurrence is not trivial on standard diagnostic MRI scans ${ }^{[13]}$.

Structural imaging methods that primarily provide anatomical information such as magnetic resonance imaging (MRI) are routinely used in the clinical setting to follow and monitor patients with brain tumour. However, they suffer from limitations in differentiating treatment necrosis from tumour recurrence. Among the non-invasive functional imaging methods which include SPECT, PET and DWI and perfusion weighted MRI; it is mainly proton MR spectroscopy that has been used in attempts to discriminate tumour from radiation necrosis. Of these methods, MR spectroscopy has advantages over PET and SPECT in that high-energy radiation is not used and radio-labelled tracers are not required. More important, MR spectroscopy is intrinsically multiparametric yielding simultaneous information about a variety of metabolites. Results of in vitro experiments have demonstrated unequivocally that the MR spectroscopic chemical profile is different for different tissues and even for different cell types $^{[14-18]}$.

The gadolinium-enhancing lesion as seen on T1 weighted MRI reflects regions where there has been a breakdown of the blood-brain barrier. This may not be a reliable indicator of active tumour due to the presence of non-enhancing tumour tissue or contrast enhancing necrosis. Typical MRI appearance of radiation necrosis is a T2hyperintense signal and T1-enhancement after contrast administration, which is difficult to distinguish from tumour progression. ${ }^{[6,8]}$

Magnetic resonance spectroscopy (MRS), a technique for non-invasive measurement of chemical substances (metabolites) in the brain has proven to be a useful tool in the evaluation of recurrent contrast-enhancing lesions in patients previously treated for primary neoplasm by helping to differentiate recurrent tumour from radiation necrosis by means of providing metabolic parameters which aids in differentiation of the same ${ }^{[7-11,18]}$. Hence, in this study we have utilised the MRS technique to evaluate the 
radiological response in high grade glioma patients receiving post-operative adjuvant therapy.

\section{Materials and Methods}

\section{Eligibility criteria}

The study protocol and consent procedure were approved by the medical ethics committee. Patients aged between 18-70 years with Karnofsky Performance Score (KPS) of more than or equal to 70 with histologically confirmed high grade glioma, with no prior history of chemotherapy and cranial radiotherapy, were eligible.

After obtaining informed consent, patients were randomly assigned to post-operative partial brain irradiation via 3DCRT technique (3DCRT arm) or IMRT technique (IMRT arm), combined with concurrent and adjuvant temozolomide (TMZ) in both the arms.

\section{Radiation therapy (RT)}

Target Volumes: Gross tumour volume (GTV) was defined as the resection cavity with any residual contrast-enhancing tissue on T1-weighted magnetic resonance imaging. The clinical target volume (CTV) was defined as the GTV with a $2 \mathrm{~cm}$ expansion to include any potential subclinical disease, with attempts made to respect the natural anatomic boundaries and organs at risk (OAR). An initial planning target volume (PTV) was generated by adding a $5 \mathrm{~mm}$ expansion to the CTV to account for setup uncertainties and a dose of $50 \mathrm{~Gy}$ in 25 fractions was prescribed to this volume. Sequential boost dose of $10 \mathrm{~Gy}$ in 5 fractions was delivered to the boost PTV which was generated by adding $5 \mathrm{~mm}$ margin to the GTV.

\section{Chemotherapy}

Concurrent Phase: Oral tablet of Temozolomide, $75 \mathrm{mg} / \mathrm{m}^{2}, 5$ days a week, was given to patients in both arms, half an hour before radiation therapy, under fasting condition.

Adjuvant Phase: Oral tablet of Temozolomide, $150-200 \mathrm{mg} / \mathrm{m}^{2}$, under fasting condition for 5 consecutive days, once in 28 days for 6 months starting after a gap of 28 days from the day of completion of concurrent chemoradiation.

\section{Radiological Response Assessment:}

Patients in both groups underwent MRI with MRS at 0,4 th and 14 th week of radiation therapy and Cho:NAA ratios were recorded.

Follow up: Patients were on follow-up till 6 months post chemoradiation.

\section{Results}

The information collected regarding all the selected cases were recorded in a Master Chart. Data analysis was done with the help of computer using SPSS (v16.0, USA).

Independent ' $\mathrm{t}$ ' test was used to test the significance of difference between continuous variables and Fisher's exact test for categorical variables. A 'p' value less than 0.05 is taken to denote significant relationship.

\section{Patient characteristics:}

Table 1: Age, gender distribution of patients and tumour laterality

\begin{tabular}{|c|c|c|c|c|c|c|}
\hline & & \multicolumn{2}{|c|}{ 3DCRT } & \multicolumn{2}{|c|}{ IMRT } & \multirow[t]{2}{*}{ P value } \\
\hline & & Number & Percentage & Number & Percentage & \\
\hline \multirow[t]{6}{*}{ Age (years) } & $21-30$ & 1 & 10 & 4 & 40 & \multirow{5}{*}{0.5} \\
\hline & $31-40$ & 2 & 20 & 2 & 20 & \\
\hline & $41-50$ & 2 & 20 & 2 & 20 & \\
\hline & $51-60$ & 4 & 40 & 1 & 10 & \\
\hline & $61-70$ & 1 & 10 & 1 & 10 & \\
\hline & Mean \pm SD & \multicolumn{2}{|c|}{$47.9 \pm 12.9$} & \multicolumn{2}{|c|}{$39.4 \pm 15.3$} & \\
\hline \multirow{2}{*}{ Gender } & Female & 3 & 30 & 5 & 50 & \multirow[b]{2}{*}{0.36} \\
\hline & Male & 7 & 70 & 5 & 50 & \\
\hline \multirow[t]{3}{*}{ Tumour laterality } & Right & 2 & 20 & 4 & 40 & \multirow[b]{2}{*}{0.33} \\
\hline & Left & 8 & 80 & 6 & 60 & \\
\hline & Total & 10 & 100 & 10 & 100 & \\
\hline
\end{tabular}


Table 2: Histology

\begin{tabular}{|l|c|c|c|c|c|}
\hline & \multicolumn{2}{|c|}{ 3DCRT } & \multicolumn{2}{c|}{ IMRT } & p-value \\
\hline Histology & Number & Percentage & Number & Percentage & \\
\hline Anaplastic Astrocytoma Gr III & 2 & 20 & 1 & 10 & \multirow{2}{*}{0.57} \\
\hline Anaplastic Oligoastrocytoma Gr III & 3 & 30 & 2 & 20 & \\
\hline Anaplastic Oligodendroglioma Gr III & 2 & 20 & 5 & 50 & \\
\hline GBM Gr IV & 3 & 30 & 2 & 20 & \\
\hline Total & 10 & 100 & 10 & 100 & \\
\hline
\end{tabular}

Table 3: Cho:NAA ratio obtained by MRS done at 14th week

\begin{tabular}{|l|l|l|l|l|}
\hline Post RT MRS Cho:NAA ratio & 3DCRT & IMRT & Total & p-value \\
\hline Less than 1.8 & $8(47.1 \%)$ & $9(52.9 \%)$ & 17 & \multirow{2}{*}{0.53} \\
\cline { 1 - 4 } More than 1.8 & $2(66.7 \%)$ & $1(33.3 \%)$ & 3 & \\
\cline { 1 - 4 } Total & 10 & 10 & 20 & \\
\hline
\end{tabular}

\section{Discussion}

Data comparing IMRT to 3DCRT planning in brain gliomas are scarce. In a study by Shannon M.Mac Donald ${ }^{[19]}$, who compared Intensity modulated radiation therapy versus threedimensional conformal radiation therapy for the treatment of high grade glioma, target dose coverage was improved with IMRT planning as compared with 3D-CRT planning and dose to normal structures was concomitantly decreased. Chan et al. demonstrated that, as compared with 3D-CRT, IMRT delivered higher doses (in excess of $10 \mathrm{~Gy}$ ) to the gross tumour while respecting the same normal-tissue constraints ${ }^{[20]}$. In our study the technique of radiation therapy did not influence the radiological response to the treatment.

\section{Radiological Response}

Elmogy et al. ${ }^{[8]}$ studied 25 patients with previously treated primary intracranial tumours. All patients received radiotherapy. MRI and multivoxel MRS were performed. The volume of interest was placed over the whole area of signal alteration. The spectra were analysed for the signal intensity of choline (Cho), creatinine $(\mathrm{Cr})$ and $\mathrm{N}$-acetyl aspartate (NAA), lipid (Lip), lactate (Lac) and myo-inositol (mI). Metabolite ratios for Cho:NAA, Cho: $\mathrm{Cr}$ and NAA:Cr were calculated. There were significantly higher Cho:NAA and Cho:Cr ratios with significantly lower NAA: $\mathrm{Cr}$ ratios between lesions denoting recurrent/residual tumours and that of radiation injury $(\mathrm{p}=0.001$ for all the three ratios). The Cho:NAA and Cho: $\mathrm{Cr}$ ratios were also significantly higher in lesions denoting radiation injury than in normal-appearing brain tissue ( $\mathrm{p}=0.032$ and $\mathrm{p}=0.008$, respectively), whereas NAA:Cr ratios were insignificantly lower in lesions denoting radiation injury than the normal-appearing brain tissue $(\mathrm{p}=0.051)$. Value $>1.8$ for Cho:NAA ratio considered as an indicator for tumour.

In our study Cho:NAA ratio in the MRS scan done at 14 weeks of RT was compared between patients in both the groups. Considering a cut off of less than 1.8 as complete response, it was found that there was no statistically significant difference in radiological response between the 2 groups.

\section{Conclusion}

Based on our study we conclude that in patients diagnosed with high grade glioma treated by radiation therapy with concurrent and adjuvant temozolomide, radiation can be delivered either with 3DCRT or IMRT technique without compromise in radiological response to treatment.

Conflict of Interest: Authors have no conflict of interest to declare

\section{References}

1. Weller M. Novel diagnostic and therapeutic approaches to malignant glioma. Swiss Med Wkly.141:w13210

2. Brandes AA. State-of-the-art treatment of high-grade brain tumors. Semin Oncol. 2003 Dec; 30(6 Suppl 19):4-9. 
3. Stupp R, Mason WP, van den Bent MJ, Weller M, Fisher B, Taphoorn MJ, et al. Radiotherapy plus concomitant and adjuvant temozolomide for glioblastoma. N Engl J Med. 2005 Mar 10; 352(10):98796.

4. Bobek-Billewicz B, Stasik-Pres G, Majchrzak H, Zarudzki L. Differentiation between brain tumor recurrence and radiation injury using perfusion, diffusionweighted imaging and MR spectroscopy. Folia Neuropathol Assoc Pol Neuropathol Med Res Cent Pol Acad Sci. 2010;48(2):81-92.

5. Nieder $\mathrm{C}$ andratschke N, Price RE, Rivera $\mathrm{B}$, Ang KK. Innovative prevention strategies for radiation necrosis of the central nervous system. Anticancer Res. 2002 Apr;22(2A):1017-23.

6. Kumar AJ, Leeds NE, Fuller GN, Van Tassel P, Maor MH, Sawaya RE, et al. Malignant gliomas: MR imaging spectrum of radiation therapy and chemotherapyinduced necrosis of the brain after treatment. Radiology. 2000 Nov; 217(2):377-84.

7. Smith EA, Carlos RC, Junck LR, Tsien CI, Elias A, Sundgren PC. Developing a clinical decision model: MR spectroscopy to differentiate between recurrent tumor and radiation change in patients with new contrast-enhancing lesions. AJR Am J Roentgenol. 2009 Feb;192(2):W45-52.

8. Sabry A. Elmogy AEM. MR spectroscopy in post-treatment follows up of brain tumors. Egypt J Radiol Nucl Med. 2011;42(s 3-4):413-24.

9. Weybright $P$, Sundgren PC, Maly P, Hassan DG, Nan B, Rohrer S, et al. Differentiation between brain tumor recurrence and radiation injury using $M R$ spectroscopy. AJR Am J Roentgenol. 2005 Dec;185(6):1471-6.

10. Schlemmer H-P, Bachert P, Herfarth KK, Zuna I, Debus J, Kaick G van. Proton MR Spectroscopic Evaluation of Suspicious
Brain Lesions After Stereotactic Radiotherapy. Am J Neuroradiol. 2001 Aug 1;22(7):1316-24.

11. Schlemmer HP, Bachert P, Henze M, Buslei R, Herfarth KK, Debus J, et al. Differentiation of radiation necrosis from tumor progression using proton magnetic resonance spectroscopy. Neuroradiology. 2002 Mar;44(3):216-22.

12. Wen, P. Y., Macdonald, D. R., Reardon, D. A., Cloughesy, T. F., Sorensen, A. G., Galanis, E., ... Chang, S. M. (2010). Updated response assessment criteria for high-grade gliomas: Response assessment in neuro-oncology working group. Journal of Clinical Oncology, 28(11), 1963-1972. DOI: 10.1200/JCO.2009.26.3541

13. Magnetic Resonance Spectroscopy for Differentiating Pseudo-Progression from True Progression in GBM on Concurrent Chemoradiotherapy V. Sawlani, R. Taylor, K. Rowley, R. Redfern, J. Martin, H. Poptani

14. Kinoshita Y, Kajiwara H, Yokota A, Koga Y. Proton magnetic resonance spectroscopy of brain tumors: an in vitro study. Neurosurgery. 1994 Oct; 35(4):606-13; discussion 613-4.

15. Kuesel AC, Sutherland GR, Halliday W, Smith IC. $1 \mathrm{H}$ MRS of high grade astrocytomas: mobile lipid accumulation in necrotic tissue. NMR Biomed. 1994 May;7(3):149-55.

16. Urenjak J, Williams SR, Gadian DG, Noble M. Proton nuclear magnetic resonance spectroscopy unambiguously identifies different neural cell types. J Neurosci Off J Soc Neurosci. 1993 Mar;13(3):981-9.

17. Rabinov JD, Lee PL, Barker FG, Louis DN, Harsh GR, Cosgrove GR, et al. In vivo 3-T MR spectroscopy in the distinction of recurrent glioma versus radiation effects: initial experience. Radiology. 2002 Dec;225(3):871-9. 
18. Kaminaga T, Shirai K. Radiation-induced brain metabolic changes in the acute and early delayed phase detected with quantitative proton magnetic resonance spectroscopy. J Comput Assist Tomogr. 2005 Jun;29(3):293-7.

19. MACDONALD, Shannon $M$ et al. Intensity Modulated Radiation Therapy (IMRT) Versus Three Dimensional Conformal Radiation Therapy (3DCRT) for the Treatment of High Grade Glioma: A Dosimetric Comparison. Journal of Applied Clinical Medical Physics, [S.1.], v. 8, n. 2, apr. 2007. ISSN 15269914.

20. Chan MF, Schupak K, Burman C, et al. Comparison of intensity-modulated radiotherapy with three-dimensional conformal radiation therapy planning for glioblastoma multiforme. Med Dosim. 2003;28(4):261-265. 